ÉGYPTE

monde arabe

\section{Égypte/Monde arabe}

15-16 | 1993

Les crises soudanaises des années 80

\title{
Famine, conflits et aide internationale au Darfour,
}

1990-1992

\section{Catherine Miller}

\section{(2) OpenEdition}

12 Journals

Édition électronique

URL : https://journals.openedition.org/ema/1017

DOI : 10.4000/ema. 1017

ISSN : 2090-7273

\section{Éditeur}

CEDEJ - Centre d'études et de documentation économiques juridiques et sociales

\section{Édition imprimée}

Date de publication : 31 décembre 1993

Pagination : 71-96

ISSN : 1110-5097

\section{Référence électronique}

Catherine Miller, «Famine, conflits et aide internationale au Darfour, 1990-1992 », Égypte/Monde arabe [En ligne], 15-16 | 1993, mis en ligne le 08 juillet 2008, consulté le 07 juillet 2022. URL : http:// journals.openedition.org/ema/1017 ; DOI : https://doi.org/10.4000/ema.1017

Ce document a été généré automatiquement le 7 juillet 2022.

Tous droits réservés 


\title{
Famine, conflits et aide internationale au Darfour, 1990-1992
}

\author{
Catherine Miller
}

1 Dans cet article, je présenterai brièvement la situation au Darfour ${ }^{1}$ entre 1990 et 1992, en m'attachant plus particulièrement au rôle de l'aide humanitaire internationale. Les problèmes rencontrés au Darfour ne sont pas spécifiques à cette province et se retrouvent dans d'autres régions du Soudan, pour ne pas dire d'autres pays de la Corne de l'Afrique ou du Sahel. Aussi les questions posées ici rejoignent-elles une problématique plus vaste: celle de la nécessité et des implications de l'aide humanitaire.

2 Les années 1990-92 furent marquées, au Darfour, par une sécheresse et une famine graves et par la recrudescence de conflits armés traduisant des luttes ethniques et politiques. Il s'ensuivit des déplacements de populations, une insécurité alimentaire et une dégradation socio-économique aiguë pour une grande partie des habitants de la province. En 1990, l'ampleur de la sécheresse provoqua la mise en œuvre d'une importante opération humanitaire au Soudan, en particulier au Kordofan et au Darfour. Cette opération, qui s'est poursuivie en partie en 1991-92 et 1992-93, se situe dans un contexte politique spécifique puisqu'elle constitue un terrain de conflit et un réel enjeu de pouvoir entre le gouvernement soudanais et la communauté internationale. Entre ces deux pôles, les populations civiles, les premières concernées, essaient de survivre et de s'adapter. 


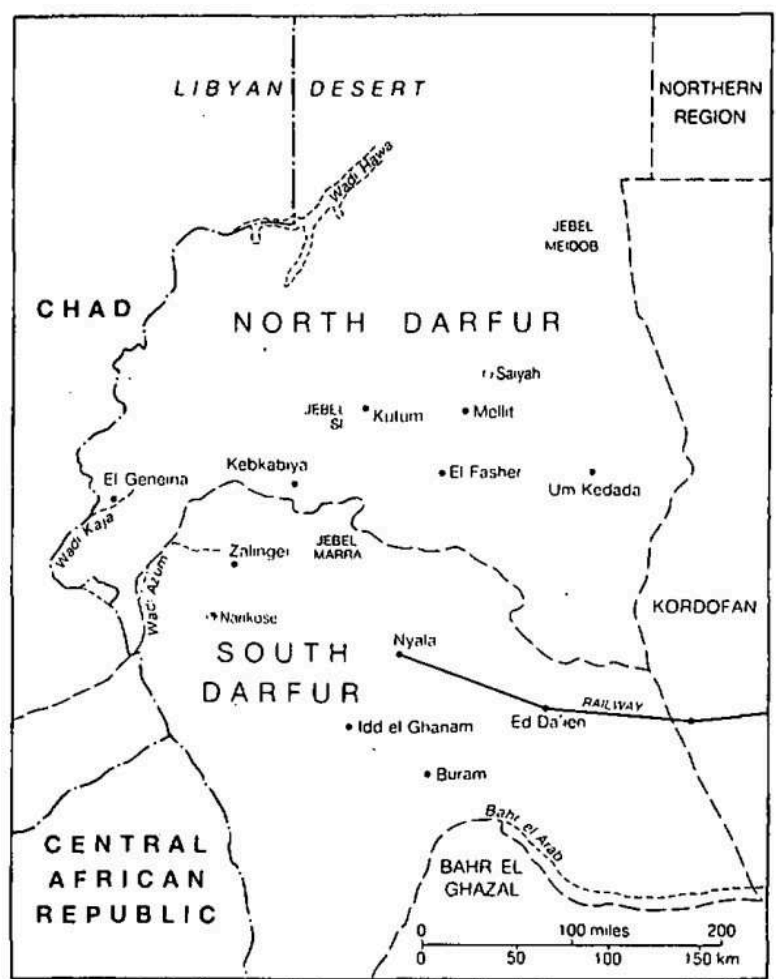

Source : A. De Wall, Famine that kills. Darfur, Sudan, 1984-85. Clarendon Press, Oxford, 1989, p. 35

Dans les pages qui vont suivre, $\mathrm{j}$ 'aborderai les thèmes suivants :

- l'impact de la sécheresse et de la famine sur les populations du Darfour (déplacement, mortalité, paupérisation), le rôle de l'aide humanitaire auprès des habitants sinistrés (évaluation, action, lacunes), l'adaptation de ces derniers à la dégradation de leurs modes de vie et l'attitude du gouvernement (central et régional);

- les causes des différents conflits armés au Darfour (différends interethniques, banditisme, ALPS, action gouvernementale, ingérences régionales), leurs conséquences et les responsabilités des différents acteurs, les liens entre la dégradation économique et les conflits armés ainsi que la relative impuissance de la communauté internationale dans les zones de conflits ;

- les contradictions et les limites de l'aide humanitaire étrangère liées au contexte politique international et soudanais (le cantonnement dans l'urgence, les restrictions d'accès aux terrains et aux populations, les choix d'aides, la manipulation des ONG et l'influence des autorités locales).

4 L'ensemble de ces questions recouvre un domaine fort vaste. Un certain nombre de points, déjà bien documentés, ne seront donc pas détaillés ici (par exemple, les causes de la sécheresse et de la désertification, la responsabilité des politiques agricoles soudanaises depuis l'indépendance, l'historique des milices) et je m'attacherai plutôt à analyser la situation actuelle au Darfour en dressant dans certains cas un parallèle avec le Kordofan, confronté à des problèmes similaires. Ce que montre l'exemple du Darfour, c'est l'extrême complexité et fragilité de l'action humanitaire prise dans les engrenages de la politique locale et internationale. Globalement, l'aide humanitaire s'est concentrée sur les populations et les régions 
touchées par la sécheresse alors qu'en 1991-92, les conflits armés furent la principale cause des déplacements de population et du déficit alimentaire. Malgré des tensions bien réelles entre le gouvernement, d'une part, et les ONG et les Nations Unies, d'autre part, un certain partage des rôles s'est mis en place dès 1991. Le gouvernement laisse de fait - et non sans de multiples restrictions - aux ONG (SCF/UK en particulier au Darfour) et aux Nations Unies (PAM, UNICEF, OMS) l'assistance humanitaire et médicale dans les zones sinistrées par la sécheresse et concentre tous ses moyens dans l'action militaire pour assurer son contrôle dans des régions charnières fermées à toute présence internationale, et sur des populations considérées comme hostiles (particulièrement au sud du Kordofan et au sud du Darfour). Dans les zones de sécheresse, l'aide humanitaire, confrontée à ses limites internes ( $c f$. infra), est loin d'avoir l'efficacité requise. Plusieurs questions restent ouvertes: les populations du Darfour ont-elles réellement besoin de l'aide humanitaire, et sous quelle forme (alimentaire, médicale, développement rural, etc.) ? Dans quelles conditions et pour quelles raisons le gouvernement (régional et central) délègue-t-il la prise en charge d'une partie des services sociaux à la communauté internationale, alors qu'il prône par ailleurs une idéologie d'indépendance et d'autosuffisance?

La situation alimentaire au Nord-DarfourSécheresse et famines

6 La famine de 1990-91, comme celle de 1984-85, s'inscrit dans un processus de désertification progressive qui pose à long terme le problème des possibilités de survie dans les régions les plus touchées du Nord-Darfour.

Sécheresse et production agricole

7 Les régions du Nord-Darfour et du Nord-Kordofan sont confrontées à une sécheresse quasi-endémique depuis la fin des années $60^{2}$. A l'exception de quelques rares bonnes années, cette sécheresse se traduit par un déclin constant de la production des zones d'agriculture pluviale et par un déficit alimentaire au niveau local ${ }^{3}$. Pour l'ensemble du Nord-Darfour, de Waal (1989: 114-119) montre que «depuis 1973, la production agricole ne couvre plus les besoins de la consommation locale ", alors même que la superficie des terres cultivées a augmenté. Selon F. Ibrahim (1984:111), la production locale couvre à peine plus de $30 \%$ des besoins. Dans les meilleures années (1988), la production n'excède pas $50 \%$ des besoins pour l'ensemble du Nord-Darfour. Dans les pires années, la production du Nord-Darfour n'a couvert que $18 \%$ des besoins en $1984-85^{4}, 15 \%$ des besoins en $1990-91$ et $30 \%$ en 1991-92 . Ces chiffres globaux masquent la disparité régionale et ne tiennent pas compte du rééquilibrage qui s'établit en temps normal entre le Nord et le Sud-Darfour, plus rarement touché par la sécheresse. Cependant, ils indiquent que le maintien d'une agriculture de subsistance dans plusieurs régions du Nord-Darfour est de plus en plus problématique. Les années de sécheresse extrême ou de famine ne font qu'accentuer l'ampleur des problèmes.

La famine de 1984

8 Les années 1984-85 virent l'apparition, au Soudan, d'une famine sans précédent au XXe siècle $^{6}$. Au Darfour, cette famine causa directement ou indirectement la mort d'environ 100.000 personnes et la disparition de $40 \%$ du cheptel - jusqu'à $70 \%$ dans les régions $\mathrm{du}$ nord les plus touchées ${ }^{7}$. Globalement, elle eut deux impacts importants: elle accéléra le processus de paupérisation du secteur agro-pastoral traditionnel et renforça l'impératif, pour les populations concernées, de rechercher d'autres sources de revenus, en particulier par le recours au travail salarié et à la migration (temporelle ou permanente). Elle provoqua, pour la première fois dans l'histoire du Darfour, 
l'intervention de la communauté internationale et l'implantation, entre 1985 et 1987, de nombreuses ONG (SCF/UK. MSF, GOAL, OXFAM. SPC, etc.). A partir de 1986, le Darfour (comme le Kordofan) devint un des terrains de prédilection de l'assistance humanitaire avec la mise en place de structures institutionnelles (le RRC, Relief and Rehabilitation Committee) et l'établissement de systèmes d'observation «précoce » (EWS, Early Warning System).

9 «Cette assistance humanitaire se concentra d'abord sur l'aide alimentaire (1985-87) puis, à partir de 1988, s'orienta vers des projets de réhabilitation et de développement $»^{8}$. Mais les moyens financiers restèrent dérisoires par rapport à l'ampleur des problèmes, et l'état d'insécurité qui prévalait dans de nombreuses régions rendait aléatoire l'implantation réelle de ces programmes. Enfin, le coup d'État de 1989 provoqua la suspension de nombreux programmes d'aide au développement, en particulier ceux financés par l'USAID conformément à l'application du décret $513 \mathrm{du}$ Foreign Assistance Appropriation Act par les États-Unis. Ni les projets du gouvernement ni les programmes d'aide n'eurent donc l'impact espéré. La sécheresse restait chronique dans les régions les plus septentrionales du Darfour (en particulier dans les districts de Mellit, Malha, Dar Zaghawa, Um Keddada) habitées par des populations agro-pastorales fragilisées.

La famine de 1990

10 Par bien des aspects, les événements de 1990-91 furent analogues à ceux de 1984-85: sécheresse très grave annoncée dès le mois d'août 1990, refus du gouvernement de reconnaître l'ampleur de la catastrophe et de faire appel à l'aide internationale, mise en place tardive de cette aide qui n'arrivera au Darfour qu'en septembre 1991 (soit un an après le commencement de la sécheresse) et ne deviendra réellement effective qu'à partir de décembre 1991-janvier 1992. Mais à l'inverse de 1984-85, la famine de 1990-91 n'a pas provoqué de phénomène migratoire massif ni d'hécatombe humaine. Les taux de mortalité restent inconnus du fait de la censure gouvernementale, les estimations officielles n'excédant pas 2.000 personnes ${ }^{9}$. Le décalage entre les déclarations alarmistes des organisations internationales en 1990 et le taux de mortalité permet de poser deux hypothèses :

- les estimations des Nations Unies seraient surévaluées, voir fausses ;

- les populations auraient mieux réagi que prévu et disposeraient de stratégies d'adaptation qui ont été sous-évaluées par les organismes internationaux.

Plusieurs remarques peuvent être faites à ce sujet. Il est sûr que les estimations des Nations Unies sont biaisées puisqu'elles reposent sur un calcul sommaire qui présuppose l'autosuffisance alimentaire (consommation annuelle par tête d'habitant production agricole $=$ déficit alimentaire), alors que, depuis longtemps, les populations ont des ressources alternatives. Cependant, les stratégies de survie des populations (que je détaillerai plus loin) ne doivent pas à leur tour être surestimées. Si elles permettent aux populations de survivre à court terme, elles ont souvent, à long terme, un impact grave sur l'environnement. Enfin, les taux de mortalité ne sont pas des indicateurs fiables pour évaluer l'ampleur d'une famine. De Waal (1989) a montré qu'en 1985, la mortalité était liée davantage aux conditions sanitaires et à la concentration de population dans les camps qu'à la famine proprement dite. C'est pourquoi le faible taux de mortalité ne doit pas faire sous-estimer l'ampleur de la crise. 
En faits bruts, la sécheresse de 1990 s'est traduite par :

- une production agricole quasi inexistante dans le Nord-Darfour et faible dans le Sud-Darfour traditionnellement région de surplus agricole, avec une moyenne de 60-70\% de déficit agricole pour l'ensemble du Darfour;

- une inflation considérable du prix des céréales sur le marché tout au long du premier semestre 1991 (le sac de millet passant de 300 livres en 1989-90 à plus de 3.000 livres à partir de mars 1991), inflation due à l'épuisement des stocks ;

- un manque de semences hypothéquant la saison 1991-92, le grain normalement gardé pour les semences étant consommé ;

- l'abandon des cultures « commerciales » (arachides, sésame, etc.) au profit de la quasimonoculture du millet ou du sorgho;

- la perte de 30 à $40 \%$ du cheptel dans le Nord-Darfour ;

- l'effondrement des cours du bétail suite à la vente forcée (distress sale) d'animaux pour couvrir l'achat des céréales, le rapport millet/mouton passant de 1/2 (300 LS/600 LS) en juin 1990 à 10/1 (3.000 LS/300 LS) en juillet $1991^{10}$. Or, la perte de ressources animales prive les populations d'un des mécanismes essentiels d'ajustement (vente d'un animal pendant les périodes de soudure);

- d'importants problèmes de réserve d'eau avec l'assèchement des hafir et la nécessité, dans certains villages, de marcher pendant près de quinze heures aller-retour pour chercher de l'eau (le manque d'eau étant la principale cause de déplacement, plus que la famine proprement dite) ;

- des taux de malnutrition élevés - près de $20 \%$ de malnutrition dans l'ensemble du NordDarfour ${ }^{11}$;

- des déplacements de populations entre octobre 1990 et juin 1991 vers les principales villesal-Fashir, Mellit, al-Geneina, Nyala ${ }^{12}$.

Cette situation se poursuivit sous une forme atténuée en 1991-92. La pluviométrie fut meilleure dans le Sud-Darfour mais la sécheresse continua à sévir dans l'ensemble du Nord-Darfour. Le déficit agricole fut estimé à $40 \%$ dans le sud et $70 \%$ dans le nord ${ }^{13}$. Les populations du nord ne pouvaient donc pas recréer leurs stocks alimentaires et, en décembre 1991, les Nations Unies lançaient un appel pour $90.000 \mathrm{t}$ d'aide alimentaire. Les troupeaux se reconstituaient en partie grâce à l'amélioration des pâturages mais cette reconstitution se faisait aux bénéfices des plus riches et au détriment des familles les plus pauvres. Les enquêtes effectuées en 1991-92 par SCF/UK dans les régions de Mellit, Um Keddada et al-Fashir montraient que, suite à la mortalité et aux ventes forcées d'animaux, $80 \%$ des familles ne possédaient plus que 3 chèvres et un âne ${ }^{14}$. L'indice des prix indiquait une stabilisation du cours des céréales (entre 800 LS dans le sud et 1.600 LS dans le nord pour un sac de millet) et une augmentation du prix du bétail (environ 2.000-2.500 LS pour un mouton). Les taux de malnutrition se stabilisaient, eux, autour de $15 \%$ et les problèmes d'eau demeuraient une des contraintes majeures pour la survie des populations, en particulier dans les régions de Um Keddada et du nord-est d'al-Fashir. Le Nord-Darfour (comme le Nord- Kordofan) s'installait donc dans une situation de crise et de déficit alimentaire chronique qui allait se poursuivre en 1992-93.

Réponses internationale et gouvernementale

famine de 1990 et la mise en place d'une opération humanitaire internationale donnèrent lieu à une partie de bras de fer entre le gouvernement soudanais et la communauté occidentale, largement décrite dans la presse de l'époque et sur laquelle 
je ne m'étendrai pas. Le gouvernement central refusa de reconnaître officiellement l'ampleur du déficit alimentaire pour l'ensemble du Soudan mais, incapable de pouvoir faire face à la situation ${ }^{15}$, il laissa aux autorités locales (gouverneurs, préfets) la responsabilité d'évaluer les besoins et de faire appel au PAM. Au niveau local; cette réticence gouvernementale se répercuta plus ou moins selon les régions et la personnalité des gouverneurs. La comparaison entre le Darfour et le Kordofan pour les années 1991-92 illustre le rôle que peuvent jouer les autorités locales.

Ainsi le Kordofan, sous la responsabilité de son gouverneur Faysal Medani, fut la première région à reconnaître officiellement l'ampleur de la famine, à demander une aide internationale et à créer en août 1990 une structure institutionnelle locale, le KDROG (Kordofan Drought Relief Operation Group), véritable instance de décision dans laquelle étaient représentés les partenaires gouvernementaux et associatifs ${ }^{16}$. Ce dynamisme local et les liens privilégiés qu'entretenaient les deux principales ONG (CARE et SCF/US) avec l'USAID permirent au Nord-Kordofan de se voir attribuer $219.200 \mathrm{t}$ de vivres pour l'année 1991. En septembre 1991, 112,516 t avaient été distribuées dans les «village councils». En dehors des problèmes inhérents à ce genre d'opération (détournement de certains convois, taxation indue des villageois par la «mafia» des transporteurs, marginalisation des nomades éloignés des points de distribution, etc.), l'aide alimentaire et les autres projets de réhabilitation ${ }^{17}$ furent relativement efficaces au Nord-Kordofan (en particulier dans les provinces de Sheikan, Bara et al-Nuhud) et contribuèrent très certainement à stabiliser les prix et les populations.

16 La situation fut toute différente au Darfour, où les autorités régionales refusaient de reconnaître l'ampleur de la famine et se montraient particulièrement peu coopératives. Les structures institutionnelles locales visant à la supervision de l'aide humanitaire (Drought and Relief Committees) restaient politiquement faibles et inaptes à organiser un système de contrôle efficace. Jusqu'en janvier 1992, les priorités du gouvernement furent d'ordre sécuritaire ( $c f$. infra) et les ONG travaillant sur place furent confrontées à d'énormes difficultés (SCF/UK en particulier, qui eut le quasi-monopole de l'aide alimentaire au Darfour). En décembre 1990, le PAM estima les besoins alimentaires du Darfour à $270.000 \mathrm{t}$ de vivres ${ }^{18}$, dont 93.000 seront allouées en janvier 1991 par la communauté internationale, compte tenu des problèmes logistiques. Neuf mois plus tard (septembre 1991), seules 18.341 t de nourriture étaient arrivées au Darfour. Ce retard était dû à des contraintes logistiques (la situation périphérique du Darfour rendant les problèmes de transports particulièrement ardus, difficulté de trouver des sociétés de transports fiables, compétition entre les ONG CARE et SCF/UK pour attirer les transporteurs, etc.), que la principale ONG (SCF/UK) n'avait pas suffisamment prises en compte, mais également au manque de soutien du gouvernement local (qui n'hésitait pas à réquisitionner les camions ou les voitures des organisations humanitaires pour ses opérations armées et refusait de donner des autorisations de déplacement au personnel non soudanais de SCF/UK) ainsi qu'à l'état d'insécurité qui prévalait dans la région.

A partir de décembre 1992, la situation s'améliora - grâce, en partie, à l'accord passé entre le $\mathrm{PAM}$ et $\mathrm{SCF} / \mathrm{UK}$ et à l'amélioration des relations entre SCF/UK et le gouvernement ${ }^{19}$-, et l'aide humanitaire devint plus efficace, incluant distribution de vivres et de médicaments, programmes de vaccination (EPI), distribution de semences. Ceci se traduisit par une stabilisation des prix (cf. supra autour de 1.500 LS pour un sac 
de sorgho). Par contre, les programmes de réhabilitation ${ }^{20}$ se mettaient en place très difficilement alors que les projets concernant l'eau sont considérés comme la priorité absolue par les populations concernées et les autorités locales. Comme le reconnaissaient les ONG, l'aide alimentaire se faisait au détriment des programmes de réhabilitation, car il est matériellement difficile de mener conjointement ces derniers et des interventions d'urgence.

Impact et limitation de l'aide humanitaire

$18 \mathrm{Au}$ cours de la première année qui suivit la sécheresse, l'aide humanitaire ne répondit donc pas à la situation d'urgence et les populations durent se débrouiller seules. Au cours de la seconde année, on notait une réelle amélioration - qualitative et quantitative - de l'aide alimentaire et médicale d'urgence, mais la transition vers des projets de réhabilitation ou de développement s'avérait difficile. On se retrouvait donc dans une situation paradoxale où une aide d'urgence continuait à être distribuée, faute d'alternative, dans des régions en voie de désertification ( $c f$. région de Malha et $\mathrm{Um}$ Keddada en particulier), ce qui entretenait d'année en année le cercle vicieux sécheresse - déficit agricole - déficit alimentaire - recours à l'aide alimentaire.

L'aide humanitaire sous toutes ses formes, au Nord-Darfour comme au Nord-Kordofan, maintient les populations sur place puisqu'elle est distribuée au niveau des «village councils ». Elle stabilise les prix, réduit l'endettement des familles et ralentit les ventes d'animaux. Par le biais des distributions alimentaires gratuites ou des programmes de Food for Work, elle s'inscrit peu à peu comme une ressource parmi d'autres pour les populations et peut donc être considérée comme faisant partie de la panoplie des stratégies de survie de ces populations. En maintenant les populations (du moins une partie) sur place, elle s'inscrit dans la logique gouvernementale qui cherche par tous les moyens à stopper le flot de migration vers les grands centres urbains. Mais à long terme, elle ne résout aucun des problèmes de fond et ne fait qu'accroître la dépendance des populations concernées.

Face à la détérioration des services sociaux, l'aide humanitaire tend à se substituer à l'État, en particulier dans le domaine médical. Ainsi, en 1991-92, les seuls médicaments disponibles au Darfour provenaient des ONG (SCF, Oxfam, Lower Saxony, GTZ) ou de l'UNICEF. SCF/UK distribuait des médicaments (kit d'urgence) dans tous les dispensaires des régions où elle travaillait (al-Fashir, Mellit, Kutum, Um Keddada, Kebkabiya, Nyala).

21 Mais cette aide médicale est liée au programme d'aide alimentaire : qu'il vienne à être interrompu, et le programme sanitaire est également suspendu. De même, en cas de conflit avec le gouvernement, les programmes peuvent cesser brutalement, comme cela a été le cas avec MSF/Belgique au Darfour. On voit donc se développer un système de protection sociale qui, tout en étant financé par la communauté internationale et mis en œuvre par des ONG, reste tributaire du contexte politique et des modes de financement (priorité à l'urgence). Ce système ne constitue en aucun cas une alternative durable aux énormes problèmes de santé et de protection sociale. Il est source de nombreux conflits entre les autorités locales et les ONG, puisqu'il est perçu comme une ingérence extérieure et un moyen de pression. Ce sentiment d'animosité est accru par la disproportion entre les moyens matériels des autorités locales et ceux des organisations internationales (le parc automobile d'al-Fashir était constitué presque exclusivement de voitures appartenant aux organisations. Du coup, le gouvernement réquisitionnait d'office des véhicules). 
L'aide internationale se retrouve donc prise au piège de ses propres limites et à la perception qu'en ont les autorités locales :

- priorité donnée au financement de l'urgence, qui oblige les ONG à inscrire toutes leurs activités dans ce cadre ;

- fragilité de cette aide qui peut être suspendue à tout moment pour raisons économiques ou politiques.

Dans ce contexte, même si elle tend à jouer un rôle croissant, elle reste une solution au coup par coup et ne résout en aucun cas l'ensemble des problèmes auxquels sont confrontées les populations.

Stratégies d'adaptation

Nous avons vu qu'au Darfour, ce n'est ni l'aide humanitaire ni l'action gouvernementale qui aidèrent les populations à surmonter la première année de famine. Ces dernières réussirent à survivre par leurs propres moyens en développant des stratégies de survie (coping mechanisms, dans le jargon humanitaire). Pour certains observateurs, ces stratégies illustrent le passage d'une économie de subsistance vers une économie monétarisée. Pour d'autres, comme de Waal, elles illustrent la volonté des populations de préserver leur mode de vie (de Waal, 1989). Sous le terme de "coping mechanisms", l'on trouve toute une panoplie de stratégies plus ou moins efficaces à moyen terme et plus ou moins nuisibles pour l'environnement. Je distinguerai ici deux types de stratégies : celles utilisées depuis plusieurs années par les populations confrontées à une sécheresse chronique, et celles utilisées dans les périodes de crise et de famine aiguës. En période de crise, une partie des stratégies "routinières" s'avèrent inefficaces car reprises par des groupes trop nombreux (recherche d'un travail salarié, par exemple).

Stratégies des « temps ordinaires »

Ces-stratégies s'organisent dans deux directions. D'une part, l'on tente d'adapter les pratiques agricoles aux conditions climatiques; d'autre part, l'on recourt de:manière croissante à des activités de type commercial ou salarié. Ces deux sortes de stratégies peuvent être complémentaires dans les régions où les populations gardent l'espoir de cultiver leur terre. Mais dans les régions les plus touchées par la sécheresse (Dar Zaghawa, Dar Gimir), les activités de type commercial supplantent peu à peu l'agriculture.

Agriculture :

Pour la culture pluviale, on note la multiplication et la dispersion des parcelles pour capter la moindre variation de pluviométrie, mais également une orientation vers la monoculture du mil ou du sorgho, qui constitue la nourriture de base, et l'abandon des cultures commerciales (arachide, sésame). Cet abandon s'explique par la faiblesse des rendements et la nécessité de cultiver des surfaces de plus en plus grandes pour espérer récolter de quoi se nourrir quelques mois ${ }^{21}$. La monoculture du mil dans les goz (plaines et dunes sablonneuses) est considérée comme un facteur supplémentaire de désertification ${ }^{22}$ puisqu'elle s'accompagne d'un déboisement systématique.

On note également une utilisation accrue des terres irrigables disponibles près des cours d'eau et le développement de la culture maraîchère. Cette dernière reste pour le moment circonscrite à des sites spécifiques ( $c f$. Kutum, Kabkabiya) sous l'impulsion le plus souvent d'ONG (cf.GTZ, OXFAM), mais ces pratiques sont par ailleurs encouragées 
par le gouvernement et de nombreux villages essaient de développer le creusement de puits en sollicitant au passage l'aide gouvernementale ou internationale ${ }^{23}$.

Activités commerciales :

Pour pallier le manque à gagner de la production agricole, les populations développent des activités commerciales et artisanales en tout genre (en particulier, collecte et vente de bois, charbon de bois, d'herbe sèche, etc.). Ces activités, qui étaient considérées comme réservées aux plus pauvres (low status trade, cf. de Waal, 1989 :143) sont maintenant reprises par un nombre croissant de villageois ${ }^{24}$ et constituent une des principales sources de revenus. Là encore, la collecte et la fabrication de charbon de bois représentent un facteur de désertification.

Sur une tout autre échelle, certains groupes ( $c f$. les Zaghawa) se sont reconvertis dans le commerce à longue distance entre la Libye et le reste du Soudan (cf. le Suq Libya à Omdurman).

Migration et travail salarié :

30 Les populations du Darfour pratiquent, depuis l'époque coloniale, une migration masculine saisonnière (ou quasi permanente), vers la région centrale pour se louer comme travailleurs agricoles. Depuis les années 70 s'est développée une migration masculine ${ }^{25}$ vers les villes ou à l'étranger (Libye principalement) et une migration familiale saisonnière vers le sud du Darfour. Là encore, le travail salarié ou le métayage (crop sharing), considérés comme des activités inférieures ", sont repris par un nombre croissant de villageois qui n'ont pas d'autre « alternative. En temps de famine, on note un sureffectif de travailleurs salariés et une baisse consécutive des salaires. De Wall (1989) insiste sur le fait que la recherche d'un emploi salarié ne conduit pas à l'abandon de l'entretien des parcelles familiales, et que les migrants retournent systématiquement dans leurs villages à la saison des pluies. En enquêtant dans des quartiers de migrants à Khartoum, j'ai pu également constater que de nombreux migrants urbains retournaient au Darfour pour aider leur famille à la période des semences. Cependant, dans les régions les plus touchées par la désertification (cf. Dar Zaghawa, Dar Gimir) on note, depuis la fin des années 70, l'abandon croissant des villages et une tentative d'installation permanente au Sud-Darfour - région de Nyala et de al-Da'in ${ }^{26}$, sud du Dar Masalit.

Stratégies des «temps de crise »

31 En cas de faillite absolue de la production agricole, une partie de ces stratégies est maintenue ou même accrue (collecte de charbon de bois). Certaines stratégies s'avèrent inefficaces ( $c f$. la vente d'animaux provoque l'écroulement des cours) et, pour survivre, les populations ont recours à des mesures plus draconiennes, changeant par exemple leur mode d'alimentation (passage à un repas par jour, collecte de baies sauvages comme le mukheil (bosca senegalensis) au nord, ou le kureb (aristida pubifolia) au sud), ou vendant des biens personnels (bijoux, sacs, tapis, etc.). Dans les villages, la solidarité s'organise : mise en commun des réserves alimentaires et prise de repas collectifs dara -, distribution d'animaux chez les populations pastorales, accueil de parents dans les villages moins touchés.

Le déplacement familial à la périphérie des villes, dans l'intention de trouver n'importe quel travail salarié (portefaix, employé de maison, porteur d'eau, etc.) apparaît comme l'ultime étape. En effet, le déplacement vers les villes peut provoquer l'éclatement des familles, les personnes âgées étant laissées au village. Du reste, certaines de ces stratégies de crise mènent à un appauvrissement supplémentaire puisqu'elles 
impliquent la vente des biens (animaux, bijoux, etc.). Nous ne disposons pas de données fiables sur l'importance qu'a pu avoir le crédit (sous forme de shail par exemple) en 1991-9:2 et sur les conséquences qu'a pu avoir l'endettement pour une certaine couche de la population (assiste-t-on à des phénomènes de dépossession, d'hypothèque. etc.?). Ce qui se dégage globalement, c'est une dynamique à plusieurs niveaux :

Dans un premier temps, les populations essaient de préserver leur mode de vie en essayant de trouver des alternatives économiques (salariat, migration saisonnière, petit commerce...) qui leur permettent tout à la fois de subvenir à leurs besoins et de pallier le déficit agricole et les pertes de cheptel sans abandonner leur région d'origine. C'est le schéma dominant depuis les années 70 dans la plupart des régions du Nord-Darfour et du Nord-Kordofan, ce qui traduit effectivement une évolution vers une économie de plus en plus monétarisée.

Dans un deuxième temps, ces alternatives occupent une place de plus en plus importante, pouvant mener jusqu'à l'abandon définitif des types de production traditionnels, abandon se traduisant le plus souvent par une migration en secteur urbain. Cette reconversion socio-économique ne se fait pas avec le même succès pour tous les individus. Il y a des reconversions réussies (celle des Zaghawas dans le commerce, par exemple), mais il y a également un grand nombre de "laissés pour compte » qui forment maintenant les classes urbaines pauvres totalement tributaires d'un éventuel travail salarié. Une autre stratégie consiste à "migrer vers le sud », ce qui fut pratiqué également par les Zaghawas dans les années 70. L'histoire du Darfour est faite de ces migrations et déplacements de populations. On sait, sans connaître les chiffres exacts, qu'une partie de la population du Nord-Darfour (pasteurs et agriculteurs) descend irrésistiblement vers le Sud-Darfour. Ce processus de « descente » vers le sud nécessiterait des recherches complémentaires pour connaître l'ampleur du phénomène, et les variations selon les groupes et les modes de propriété foncière ${ }^{27}$, les contraintes liées à la surpopulation. Il semble qu'actuellement cette stratégie « historique » se heurte à des problèmes politiques croissants.

Face à la désertification et en l'absence d'intervention extérieure (gouvernement, communauté internationale), les stratégies de survie ne constituent pas une solution économique viable à long terme dans la région d'origine. Il est frappant de constater que dans les régions antérieurement touchées par la sécheresse (Dar Zaghawa), à une époque où l'aide internationale n'existait pas encore, la solution ultime fut la migration, la reconversion dans le commerce et le quasi-abandon de la région d'origine.

En circulant, tout au long de l'année 1991-92 au Nord-Darfour (en particulier dans les régions de Mellit, Um Keddada et nord-est d'al-Fashir) la question qui venait spontanément aux lèvres était : "Comment et pourquoi les populations s'obstinentelles à rester là ?» Que se serait-il passé si l'aide internationale n'était pas intervenue en 1991 ? Est-il de bonne politique d'essayer de maintenir ces populations coûte que coûte dans ces régions désolées ? De fait, il est sûr que les migrations représentent un phénomène croissant au Darfour, mais les flux migratoires se heurtent à des contraintes également croissantes : manque de travail, que ce soit en Libye ou dans les grandes villes du Centre-Soudan, surpopulation au Sud-Darfour et compétition accrue pour avoir accès aux terres (que ce soit sous forme de pâturage ou de terres cultivables). Les troubles ethniques qui ont ensanglanté le Darfour en 1990, 1991 et 1992 sont une illustration des contraintes qui menacent les migrants potentiels.

Conflits armés et insécurité 
37 Les années 1990-91 ont vu la recrudescence au Darfour de conflits armés dont les origines diverses reflètent à la fois des rivalités interethniques traditionnelles exacerbées par le contexte économique actuel, et des rivalités politico-militaires traduisant les différentes luttes pour le pouvoir (ingérence libyenne et tchadienne ${ }^{28}$, tentatives de l'État ou de certains partis pour renforcer leur contrôle, lutte contre l'ALPS, etc.). En 1991-92, les principaux conflits ont été :

- les combats entre groupes ethniques ou milices «tribales » (principalement arabe/four mais également entre groupes pastoraux Zaghawa/Rezeigat, Habbaniya/Fellata...) ;

- l'action gouvernementale contre le « banditisme »;

- le conflit ALPS/armée.

On estime qu'au total plus de 100.000 personnes « déplacées » ont dû fuir leurs villages à la suite de ces conflits ${ }^{29}$ entre 1991-92, principalement dans les régions de Zalingey, Jebel Marra, Wadi Salih, Buram, Kabkabiya. A ce chiffre s'ajoutent les déplacés sudistes (principalement Dinka) du Bahr al-Ghazal estimés à près de 70.000 en juin 1992 dans les provinces d'al-Da'in et Nyala. En 1991-92, les conflits armés provoquèrent un déficit agricole de près de $50 \%$ dans les régions les plus productives (Zalingey, Wadi Salih).

Conflits ethniques et milices « tribales » Conflits ethniques

39 Les conflits ethniques, principalement entre sédentaires et semi-nomades, ont toujours prévalu au Darfour, de même que les conflits entre semi-nomades, plus particulièrement en période de sécheresse où la compétition pour l'eau et les pâturages s'intensifie. Une des principales causes de conflit concerne la délimitation spatiale et temporelle des routes de transhumance empruntées par chaque groupe pastoral. Pendant la période coloniale, les talaga laws délimitaient de façon stricte les droits de chaque groupe, sédentaire et pastoral. En cas de non-respect de ces lois, les conflits étaient réglés par les autorités locales (chefs). Les changements politiques (disparition de l'indirect rule) et climatiques ont rendu ces lois caduques et inapplicables sans qu'une nouvelle législation ait été réellement élaborée ${ }^{30}$. A ce vide Juridique s'ajoute le surarmement des groupes, dû au prolongement du conflit tchadien et à l'affaiblissement du contrôle de l'État, d'où l'instauration d'un climat d'anarchie ou règne la loi du plus fort. A la suite de la sécheresse de 1990, on notait au Darfour une pléthore de conflits armés entre pasteurs et sédentaires ou entre groupes pastoraux :

- province de Nyala : conflits entre Taisha et Gimir à Katila, entre Gimir et Fellata à Tulus, entre Rezeigat et Birgit à Muhageriya ;

- province d'al-Da'in : conflits entre Rezeigat et Zaghawa, Rezeigat et Birgit, Rezeigat, Habbaniya et Miseriya ;

- province de Wadi Salih : conflits entre Arabes et Four ;

- province de Zalingey : conflits entre Arabes et Four.

Les conflits traditionnels prennent souvent des allures de vendetta et se règlent au bout de quelques mois comme ce fut le cas à Katila en septembre 1991 entre les Taisha et les Gimir. Mais la plupart des conflits sont aggravés par l'action politico-militaire des milices, qui leur donne une tournure de guerres civiles comme c'est le cas depuis des années entre les milices arabes et les Four.

Les milices

41 Les premières milices "arabes" (murahalin) se sont développées dans les années 70 (comme aile armée du parti Umma) et ont connu un réel essor en 1986-87 sous le gouvernement de Sadiq al-Mahdi, puisqu'elles étaient utilisées par le gouvernement pour lutter contre l'ALPS et les groupes sympathisants (Dinka) à la frontière du Sud- 
Darfour (et Sud-Kordofan) et du Bahr al-Ghazal ${ }^{31}$. A ces milices "soudanaises » s'ajoutaient les groupes armés soutenus par la Libye et le Tchad dans le prolongement de la guerre tchadienne et des visées annexionnistes de la Libye (annexion du Darfour à la province de Kufra). Dès 1986, ces milices arabes/profitant de leurs liens privilégiés avec le pouvoir et soutenues activement par la Libye ${ }^{32} s^{\prime}$ en prenaient aux groupes non arabes du Darfour et aux Four en particulier, pour des motifs économiques (razzias), historiques et politiques (les Four étant considérés comme le principal obstacle à une hégémonie arabe au Darfour).

42 Entre 1986 et 1989, les conflits entre Arabes et Four firent près de 6.000 morts $^{33}$. En mars 1988, les Four créèrent des groupes d'autodéfense. Le conflit entre Four et Arabes connut une pause en juin 1989 (conférence de réconciliation) pour reprendre, de façon accrue, au printemps 1991. Près de 134 villages furent brûlés entre mars et mai 1991 dans les provinces de Zalingey et Wadi Salih, provoquant le déplacement d'environ 50.000 personnes. Les événements de 1990-91 apparaissent donc comme un prolongement de ceux de 86-88, mais cette fois, les milices non arabes se sont renforcées (création, en juin 1990, du front Four, contact plus actif entre l'ALPS et le front Four et en 1991 participation active de l'armée et du gouvernement: bombardement de villages en octobre 1991) dans le cadre d'une campagne de désarmement et de lutte contre le banditisme.

Campagne de désarmement et de lutte contre le banditisme

Tout au long de la période 1990-91, on assiste à une recrudescence des attaques à main armée perpétrées par des groupes isolés ou des fractions de milices contre les convois commerciaux ou le cheptel (chameaux notamment), provoquant la mort de nombreux civils $^{34}$ et militaires. Cet état d'insécurité entraîne un ralentissement notable des activités commerciales (nécessité de se déplacer en convois, uniquement de jour, portions de routes impraticables) et le refus des transporteurs de délivrer l'aide humanitaire.

En novembre 1990, le Darfour est placé sous loi martiale et en octobre 1991, le gouverneur du Darfour, le colonel Tayeb Ibrahim Muhammad Kheir, lance une campagne de désarmement et de lutte contre le banditisme qui se traduit, entre autres, par le bombardement de 80 villages dans l'ensemble du Darfour (plus particulièrement à la frontière tchado-soudanaise et dans les reliefs montagneux). Ces bombardements, annoncés officiellement dans la presse gouvernementale, suscitent des nombreuses critiques de la part de l'opposition, qui considère que le gouvernement applique une politique sélective en réprimant plus sévèrement certains groupes (les Four, notamment) et en favorisant les groupes arabes (en particulier les Rezeigat).

Malgré l'ampleur des moyens mis en œuvre, le banditisme prévalait toujours au NordDarfour en 1992, comme l'attestèrent les attaques perpétrées contre des voitures de $\mathrm{SCF} / \mathrm{UK}$ dans la région de Kutum. Il semble que la campagne de désarmement eut un impact très limité malgré l'optimisme de circonstance du gouvernement. Mais l'intrusion de l'ALPS au Darfour allait permettre à ce dernier d'employer les grands moyens pour réprimer le front Four et renforcer son contrôle dans les provinces du sud-ouest (Wadi Salih, Zalingey, Buram).

L'entrée de l'ALPS au Darfour

46 Le 11 novembre 1991, l'ALPS faisait une percée éclair au Darfour sous la direction d'un Four, Dawud Yahia Bolad, ancien transfuge du FNI qui aurait rejoint l'ALPS en 1990. Elle atteignit rapidement le sud du Jebel Marra, écrasant au passage deux bataillons (114 et 
224). Cette opération fut un choc pour le gouvernement, qui réagit avec la dernière vigueur (envoi de bataillons, recours à l'aviation, appel à la participation des milices arabes, consolidation des forces paramilitaires que sont les forces de défense populaire - Difa' cha'biyya-, arrestation de nombreuses personnalités four, etc.). Le 12 janvier 1992, l'ALPS était écrasée et Dawud Bolad arrêté. Selon les autorités régionales, quelque 240 villages auraient été rasés et 60.000 personnes déplacées.

La lutte contre l'ALPS permit au gouvernement de lancer officiellement le jihad tant au Darfour qu'au Kordofan. Le jihad impliquait la participation directe des populations locales, qui devaient fournir un quota de participants aux forces de défense populaire ainsi qu'une participation financière et matérielle. Le jihad incluait non seulement la lutte contre l'ALPS dans les provinces du Sud-Soudan, mais aussi la lutte contre les groupes ethniques supposés sympathisants comme les Nouba ou les Four, que ceux-ci soient musulmans ou non. Le jihad impliquait enfin une phase militaire de "pacification» (neutralisation des opposants) suivie d'une phase de "réhabilitation » avec réalisation de projets de développement. On sait qu'au Sud-Kordofan, le jihad se traduisit par le déplacement forcé des populations nouba vers des peace villages au SudKordofan et des campements au Nord-Kordofan (20.000 personnes déplacées à al-Obeid, Bara, al-Nuhud et Um Ruwaba en mai 1992).

Cette politique de déplacement forcé de population suscita de nombreuses questions et inquiétudes : s'agissait-il d'un projet national global ou d'une initiative personnelle du nouveau gouverneur du Kordotan ? Y avait-il une réelle volonté d'évacuation - voire de génocide - des populations pour s'approprier les terres, comme le soutient le front nouba, ou s'agissait-il plus simplement d'une campagne de "pacification $»^{35}$ ? Ces inquiétudes étaient partagées par les Four, qui semblaient pourtant bénéficier d'une politique différente, oscillant entre répression et tentatives de conciliation.

Conflits armés et aide humanitaire

Les années 1991-92 représentent donc un tournant important de la politique gouvernementale au Darfour. Alors que la fin du régime Nimeyri et la période de transition démocratique se caractérisaient par l'affaiblissement de l'armée et du pouvoir central face aux tendances régionalistes et aux mouvements nationalistes, l'année 1991-92 se caractérise par la volonté du gouvernement central d'imposer son contrôle et d'éliminer les forces nationalistes en privilégiant le règlement militaire sur la concertation. Les provinces du Sud-Kordofan et du Sud-Darfour sont traitées comme celle du Sud-Soudan et placées sous occupation militaire. Pendant toute la période des opérations militaires (de septembre 1991 à janvier 1992), les provinces du Sud-Darfour (Zalingey, Buram, Wadi Salih) demeureront fermées à toute présence internationale et les populations déplacées ne recevront aucune aide ${ }^{36}$ alors qu'elles sont dans une situation plus dramatique que les populations touchées par la sécheresse. Le RRC n'a aucun pouvoir d'intervention puisqu'il ne reçoit aucune demande officielle.

Peu à peu, à partir de janvier 1992, le gouvernement crée des structures parallèles (sous la férule de l'association Peace and Development de feu Abu Gisseissa) qui sont censées prendre en charge l'assistance aux personnes déplacées et les projets de réhabilitation dans les villages détruits. A l'inverse de ce qui se passe dans les zones sinistrées par la sécheresse, ces programmes ne font pas appel à l'aide internationale et doivent être mis en œuvre par des associations gouvernementales (chabab al-watan - les jeunes de la nation) ou soudanaises (SPC, IARA, Da'wa islamiyya). Mais faute de moyens, ces programmes ne sont pas opérationnels. 
51 A partir de juin 1992, on note un infléchissement de cette politique : les gouvernements du Darfour et du Kordofan demandent le soutien de la communauté internationale tout en refusant la présence d'ONG étrangères et en insistant sur le fait que l'aide doit transiter par des ONG soudanaises. Cette demande place la communauté internationale dans une situation inconfortable. D'une part, il est difficile de refuser d'aider des populations dans une situation désespérée; d'autre part, il est inconcevable de ne pas avoir un droit de regard sur les implications réelles de cette aide et sur ses objectifs à long terme. On se retrouve ainsi dans une situation paradoxale: la communauté internationale, par le truchement d'arrangements locaux non officiels, se retrouve à aider financièrement des opérations qu'elle réprouve d'un point de vue humanitaire ${ }^{37}$. $\mathrm{Au}$ Darfour, les autorités locales, soucieuses de montrer leur bonne volonté vis-à-vis des Four, se montrent légèrement plus souples, et une coopération entre le PAM-SCF/UK et SRC se met en place en août 1992 pour la région de Wadi Salih.

L'aide humanitaire apparaît comme particulièrement faible dans les régions charnières du Sud-Darfour et du Sud-Kordofan, soumise au bon vouloir des autorités régionales et centrales, et incapable d'aider les populations les plus touchées: les déplacés. Le gouvernement a compris l'enjeu formidable que peut représenter cette aide et, jouant sur les contradictions du système humanitaire, il essaie d'en faire l'instrument de sa propre politique.

GOS et aide humanitaire

Depuis 1985 , l'aide humanitaire fait partie intégrante du paysage politique soudanais (en particulier avec les opérations OLS au Sud-Soudan). Mais depuis 1989, les organisations humanitaires sont confrontées à un nouveau contexte politique puisque le GOS veut promouvoir une politique d'autosuffisance et a pour ambition de créer un homme nouveau, travailleur et productif ${ }^{38}$. Le gouvernement recherche donc une aide au développement (en particulier pour de grands projets agricoles dans la région du centre, comme l'extension du canal de Rahad) et est hostile, a priori, à l'aide humanitaire alimentaire. A l'opposé, les pays occidentaux limitent leur soutien à l'aide d'urgence, ce qui entrave le dialogue, entre les deux partenaires. Nous avons vu cependant que malgré des déclarations hostiles, en particulier dans la presse officielle, le gouvernement avait le plus souvent adopté une attitude pragmatique en laissant travailler les $\mathrm{ONG}$, sous un contrôle strict, dans les régions touchées par la sécheresse.

Les principaux bénéficiaires de l'aide internationale, en 1991-92, ont été les populations rurales du Nord, et non les populations déplacées des régions en guerre. Cela s'explique en partie par le manque de moyens de Khartoum, qui ne pouvait faire face seul à cette famine. A moyen terme, il pose cependant la question des priorités agricoles du gouvernement. N'étant pas experte en la matière, je laisserai cette question aux spécialistes. Mais il semble que, malgré ou à cause d'une idéologie "d'autosuffisance ", le gouvernement actuel néglige une fois de plus le secteur traditionnel et se concentre sur les régions où de grands projets agricoles (dont on connaît le coût écologique) sont envisageables, que ce soit dans la région centrale ou dans les plaines du Sud-Kordofan et du Sud-Darfour.

En 1991-92, le gouvernement a organisé une multitude de conférences de développement dans toutes les régions du Nord-Soudan. Chaque région était invitée à préparer un calendrier de projets économiques plus ou moins réalistes. Mais faute de capitaux, ces projets risquent pour la plupart de rester lettre morte, tandis que la vente de terres fertiles à des compagnies ou de gros propriétaires s'accélère dans les régions 
«reconquises ». L'aide humanitaire, même à petite échelle, allège donc le fardeau du gouvernement en maintenant les populations à un stade minimum de survie.

Cependant, le gouvernement ne souhaite pas maintenir sur le territoire cette présence "étrangère " et tâche de plus en plus de remplacer les organisations internationales par des organisations nationales et régionales, islamistes de préférence. C'est chose faite dans les territoires reconquis au Sud-Soudan; dans le Sud-Kordofan et dans les camps de déplacés autour de Khartoum, auxquels seules les organisations soudanaises ont droit d'accès ${ }^{39}$. Une partie d'échecs se déroule entre la communauté internationale, qui privilégie "ses $O N G$ », et le gouvernement soudanais, qui privilégie "ses associations", entraînant des tentatives de solutions intermédiaires comme le jumelage entre $\mathrm{ONG}$, le transfert de fond d'ONG internationales à ONG soudanaises, etc. Cette partie d'échecs se fait au détriment des populations concernées, surtout dans les régions de guerre civile où il semble que le gouvernement privilégie, cette fois, les nécessités idéologiques plutôt que les considérations humanitaires pragmatiques.

En 1991-92, les ONG internationales, divisées et obéissant à la loi du marché, acceptaient de travailler et de se cantonner dans les régions et les domaines où leurs interventions étaient autorisées par le gouvernement et sans trop s'interroger sur la finalité et l'avenir de leur action. Les Nations Unies acceptaient également cet état de fait et prenaient soin de distinguer aide alimentaire et droits de l'homme. Par là même, elles permettaient à l'aide humanitaire de se poursuivre, de façon plus ou moins chaotique et efficace, dans les régions "calmes", servant de palliatif à une réelle solution, et restaient impuissantes face aux désastres des conflits armés. On retrouve là un schéma désormais classique.

Conclusion

Quelles que soient ses limites, insuffisances, contraintes et manipulations, l'aide humanitaire occupe une place de plus en plus importante sur la scène politique internationale. L'exemple du Darfour montre qu'elle tend à devenir partie intégrante des systèmes économiques et sociaux même lorsque les États bénéficiaires sont idéologiquement hostiles aux ingérences extérieures. Son efficacité est plus que contestable et son impact réel difficile à évaluer. Mais elle représente une nouvelle carte, comprise par tous, et un objet de tractations, de pressions multiples entre les pays «donateurs» et les pays « receveurs». L'aide n'exclut en effet nullement les références idéologiques, comme l'atteste par exemple le foisonnement récent des ONG islamiques au Soudan.

\section{BIBLIOGRAPHIE}

Al-Arifi S. A., « Development Strategy for the Arid Zone of Western Sudan », in Y. F. Hassan \& P. Doornbos ed., The Central Bilâd al-Sûdân, p. 131-148, 1977.

Awad I. A., « The Impact of Desertification Caused Migration on Assimilation and Migrants' Urban Social Integration: a Case from Western Sudan », ICAS, Khartoum, 11-14déc.1991. 
Awad M. H. :

- «Self Sufficiency Redefined », DSRC Seminar Series Discussion Paper n 97, Khartoum, 1992.

- «The Evolution of landownership in the Sudan », The Evolution of Agrarian Relations in the Sudan, al-Fatih Shaa al-Din Ithaca Press, 1987. pp 31-56.

Al-Awad S. A., « Traditional Agriculture in the Northern District of Southern Kordofan Province ", Socio-Economic Change in the Sudan, M.H. Awad ed., Graduate College Publication $\mathrm{n}^{0}$ 6,1983, pp. 39-66.

BSIM, Baseline Survey Information Mission, sept. 1991. Nations Unies Khartoum,1991.

Borton J., Clay E., Shoham J. \& Nicholds N., « The Contribution of the Relief and Development Institute to the Evolution of the Agenda on Famine and Emergency Relief » , Disaster, vol. 15, $\mathrm{n}^{\circ}$ 4,1991. p. 373-378,

Duffield M., « From Emergency lo Social Security in Sudan », Disaster, vol. 14, n 3-4,1989, pp. $187-203$ \& 322-334.

Ibrahim F., Ecological Imbalance in the Republic of the Sudan with Reference to Desertification in Darfour, 1984. Bayreuth.

Kilgour M. C., "Refugees and Development: Dissonance in Sudan », in State and Society in Sudan, J. O. Voll ed., Indiana University Press, 1991, pp. 123-136.

Kontos S., « Farmers and the Failure of Agro-Business in Sudan » in State and Society in Sudan, J. O. Voll ed., Indiana University Press, 1991, pp. 137-162.

Prunier G., « Ecologie, structures ethniques et conflits politiques au Darfour », in Soudan Histoires, identités et idéologies, Bleuchot et al. ed., Reading, lthaca Press, 1991. pp. 85-103.

Salih A., « Development Strategy for the Semi-Desert Zone of Western Sudan », in The central Bilad al-Sudan, Tradition and Adaptation, Y.F. Hassan \& P. Doornbos eds, Khartoum Sudanese Library Series $n^{0} 11,1977$, pp. 131-148.

Tully D., Culture and Context in Sudan. The Process of Market Incorporation in Dar Masalit, State University of New-York Press, 1988.

Waal A. de. :

- «Some Comments on Militias in Contemporary Sudan », in Soudan - Histoires, identités et idéologies ; op. cit., 1991.

— Famine that kills, Oxford University Press, 1989.

Wohlmuth Karl, Sudan's National Policies on Agriculture, University of Bremen SERG, Discussion Papers, 1987.

\section{ANNEXES}

Abréviations

ALPS $=$ Armée de libération du peuple soudanais

APU = Agricultural Planning Unit (GOS)

$\mathrm{BIT}=$ Bureau international du travail (NU)

BSIM = Baseline Survey Information Mission (doc. Nations Unies sept. 1991) 
CARE $=$ ONG internationale, principalement américaine

EWS= Early Waming System

GOAL $=$ ONG irlandaise

GOS = Government of Sudan

GTZ = Agence allemande de développement

lARA = Islamic African Relief Association (Soudan)

MFEP = Ministry of Finance and Economic Planning (GOS)

MSF = Médecins sans frontières

LS = Livre soudanaise (monnaie soudanaise)

$\mathrm{NU}=$ Nations Unies

OLS = Operation Lifeline Sudan

ODA = Overseas Development Activities (GB)

OMS = Organisation mondiale de la santé (NU)

ONG = Organisation non gouvernementale

OXFAM $=$ ONG britannique

PNUD = Programme des Nations Unies pour le développement

$\mathrm{PAM}=$ Programme alimentaire mondial (NU)

RRC $=$ Relief and Rehabilitation Committee (GOS)

SCF/UK = Save The Children Fund (GB)

SCF/US = Save the Children Fund (USA)

$\mathrm{SPC}=$ Sudanese Red Crescent (Soudan)

USAID = United State Agency for International Development

\section{NOTES}

1. Superficie de $500.000 \mathrm{~km}^{2}$, population estimée entre 3 et 4 millions (3.093.699 selon le recensement de 1983 et 4.369.646 selon les sources des Nations Unies en sept. 1991).

2. Selon Fouad Ibrahim (1984 : 67), la sécheresse n'est pas un phénomène nouveau. Depuis 1919, les cycles de sécheresse et d'humidité ont alterné comme suit à al-Fashir : PériodeDuréeTypePluviométrieDéviation/norme de $286 \quad \mathrm{~mm} 1919-3921$ ansHumidité329,3 $\mathrm{mm}+15,1$ \%1940-4910ansSécheresse218,9 $\mathrm{mm} \quad-\quad 23,5 \% 1950-6516$ ansHumidité329.2 $\mathrm{mm}+15,1 \quad \% 1966-8318$ ansSécheresse201,0mm- 29.7 \%1984-939 ansSécheresse170,0mm- 40,6\%

3. La documentation sur les politiques et l'état de la production agricole est abondante. Voir par ex. : Duffield (1989), al-Arifi (1977), Fouad Ibrahim (1984), Wolmuth (1987), etc. La plupart des auteurs insistent sur le facteur démographique (augmentation de la population, intensification de l'agriculture et sur pâturage) pour expliquer la désertification.

4. Voir bibliographie ci-après. 
5. Estimations données par la cellule d'urgence des Nations Unies / Khartoum.

6. Pour un historique des famines au Darfour, voir de Waal, 1989. Les famines les plus importantes (1886-1890) furent répertoriées à la fin du siècle dernier et accompagnaient la déstabilisation de la région.

7. Cf. de Waal, 1989: 153.

8. Parmi les nombreux projets qui ont été lancés au Darfour, on nota de petits projets montés par les ONG comme des programmes agricoles (OXFAM, GT2), des programmes de creusement de puits, des programmes médicaux (MSF, GOAL) et des projets plus lourds (cf. le Darfour Rehabilitation Program) signés en 1986 entre le PNUD-BIT-FAO et le MFEP (Ministry of Finance and Economic Planning) qui sont la prolongation des projets DECARP (Desert Encroachment Control and Rehabilitation Programm 1977). Il y a également des programmes de soutien institutionnels comme l'établissement de l'APU (Agricultural Planning Unit) subventionné par l'ODA.

9. Aucune donnée sur le taux de mortalité n'était disponible au Darfour en 1990-91. Le chiffre de 2.000 personnes est une estimation publiée dans un document des Nations Unies/RRC en septembre 91 (BSIM). Le décalage entre les chiffres de 1984-85 et 1990-91 peut être en grande partie lié aux problèmes d'accès à l'information.

10. Les chiffres des Nations Unies donnent le rapport suivant entre les cours du millet et du mouton entre janvier et septembre 1991 :

ProvinceJanvierMarsJuilletSeptembreNyala1200/3503500/3504000/3502300/550AI-

Dain2100/10003000/4004500/350

2300/1200Buram2400/2003600/3003600/4002100/500Zalingei700/2001000/200.2500/2503000/300Wadi Sali500/250500/2501800/19002000/500AI-

Fashir3000/5003500/5004000/6504200/800Geneina600/500600/5001500/5002200/550Kutum1500/3502600/4003000/3803600/1200UmRuwaba3600/6003 11. Taux de malnutrition relevés par OXFAM et SCF/UK entre mars et septembre 1991 : $22 \%$ dans la province d'al-Fashir, $20 \%$ dans la province d'al-Geneina, 20-25\% dans la province de Kutum, 26,5\% dans la province d'Um Keddada. Les résultats des enquêtes de malnutrition, quoique controversés, restent le principal indicateur utilisé par les organisations. Les taux élevés de malnutrition ne coïncident pas avec le faible taux de mortalité, ce qui implique soit que ces taux sont surévalués, soit que la malnutrition ne cause pas immédiatement la mort.

12. Voici quelques estimations fournies par les Nations Unies : 18.000 déplacés dans la province d'al-Fashir en mars 1991, 16.300 dans la province de Kutum, 20.000 dans la province d'Um Keddada. Ces chiffres, souvent basés sur les estimations des administrateurs locaux, ont tendance à être surestimés (source BSIM).

13. Source BSIM.

14. Selon F. Ibrahim (1984:130), en 1977, la moyenne d'un cheptel par famille était de 10 chameaux, 7,5 vaches, 22,5 moutons et 19,5 chèvres.

15. Je manque de données pour évaluer l'action du gouvernement dans le domaine humanitaire (cf. la redistribution de la zakât, l'aide aux orphelins et aux veuves, la distribution de céréales à des prix subventionnés qui semble avoir bénéficié en priorité aux fonctionnaires).

16. Le KDROG regroupe des représentants des Ministères de l'Agriculture, des Finances, des Affaires sociales, de la Sécurité, du RRC et des ONG comme CARE, SCF/US, SRC. IARA, etc.

17. En particulier les programmes d'eau et de Food for Work entrepris par l'UNICEF, le BIT et le PNUD. 
18. Ce chiffre est calculé sur la base de deux millions de personnes "à risque » nécessitant une aide de 400 gr par personne et par jour.

19. Un accord fut passé en octobre 1991 entre le PAM et SCF/UK, le PAM s'engageant à acheminer l'aide alimentaire jusqu'au Darfour et SCF/UK s'occupant de la distribution dans les village councils.

20. En particulier un programme d'eau SUNDOS financé par le PNUD et réalisé par SRG, SCF et ILO. De même les programmes de développement intégré comme ADS (Area Development Scheme) à Um Keddada, ou SPWP (Special Public Work Programm) financé par le PNUD.

21. Les rendements par feddan étaient en 1991-92 de 0 à $1 \mathrm{sac}$ de $50 \mathrm{~kg}$ dans la région de Mellit (nord-est d'al-Fashir), et de 2 sacs dans la région de Kabkabiya (sud-ouest d'alFashir).

22. Cf. F. Ibrahim (1984: 106). Cependant, il semble que les agronomes ne soient pas unanimes sur ce point. Des études citées par Tully (1988:117-122) montrent que dans un système traditionnel, les mêmes terres peuvent être cultivées pendant près de quinze ans grâce à un processus organique de fertilisation à partir des déchets. A l'opposé, les effets de la culture d'arachide sur les sols seraient beaucoup plus hasardeux.

23. Le creusement de puits était la priorité absolue présentée par de nombreux villages pour des programmes de Food for Work.

24. En mars 1992, tous les villageois que nous avons interrogés dans la région du nordouest d'al-Fashir indiquaient que la collecte et la vente de charbon de bois représentait l'une des sources essentielles de leur revenu.

25. Cf. Tully $1988: 181$ : « Migration by one member (or more) of the household can be a strategy to maintain a rural household with a degree of autonomy ».

26. Entre 60 et $70 \%$ des Zaghawas vivraient en dehors du Dar Zaghawa, soit dans les grandes villes (al-Fashir, Khartoum).

27. Tully (1988: 210) montre qu'au Dar Masalit, suite à la limitation croissante des terres cultivables, l'accès à la terre s'est modifié. Alors qu'auparavant l'accès se faisait en allant déboiser une parcelle vierge, maintenant la plupart des terres sont héritées ou louées car occupées.

28. Ainsi, en mai 1990, l'armée tchadienne entre au Darfour pour y poursuivre les partisans d'Idriss Deby et en novembre 1990, des forces tchadiennes pénètrent à Kutum, Zalingey et al-Geneina (source : Sudan Update).

29. Source : Emergency Unit / Nations Unies / Khartoum, en sept. 91.

30. Il y a eu, en 1986, des propositions comme le Land Tenure Task Study, mais elles n'ont jamais été réellement appliquées.

31. Pour plus de détails sur l'historique des milices, voir de Waal 1991 et Prunier 1991 (bibliographie d-après).

32. G. Prunier (1991 : 94-95).

33. G. Prunier (idem).

34. Selon le gouvernement soudanais, les attaques à main armée auraient causé la mort de 1.200 personnes depuis 1984 .

35. Les dernières informations en provenance des Monts Nouba indiquent une accentuation de la répression (cf. Sudan Update, vol. 4, n², 16/3/93 et vol. 4, 31/3/93) et la mise en vente de terres au sud du Kordofan pour des projets agricoles privés.

36. La seule ONG qui travaillait au Sud-Kordofan en 1991 était le SRC (Sudanese Red Crescent) financé par La Ligue Internationale de la Croix-Rouge. Le SRC s'occupait des déplacés sudistes à Abyei. 
37. Ainsi, des accords entre les préfets de Bara et d'Um Ruwaba, d'une part, et CARE et SCF/US, d'autre part, amenèrent ces deux ONG à fournir une aide alimentaire aux déplacés nouba internés dans les camps, alors que la communauté internationale s'opposait à la création de ces camps.

38. $C f$. l'omniprésence des slogans du type « Nous consommons ce que nous produisons ».

39. II y a eu par exemple toute une polémique, en 1992, sur l'épidémie de kala-azar à Bentiu, où le gouvernement a refusé de laisser travailler MSF/Hollande et insistait pour que ce soit la Da'wa islamiyya qui combatte l'épidémie.

INDEX

Mots-clés : Soudan

\section{AUTEUR}

CATHERINE MILLER

CNRS-CEDEJ 